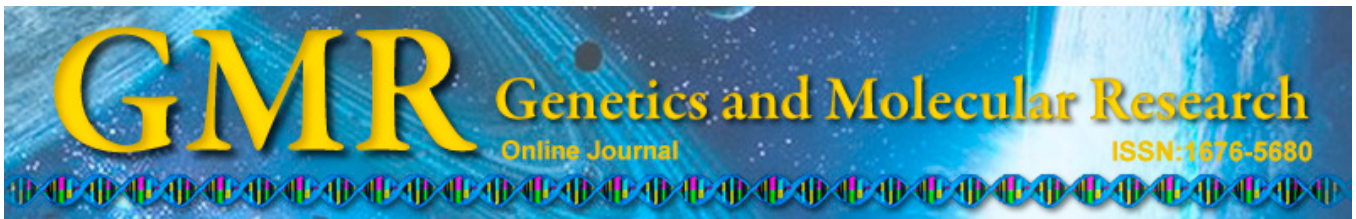

Case Report

\title{
Local skin flap with vacuum-seal drainage to facilitate healing of ACS
}

\author{
R.B. Luo ${ }^{1,2}$, Y.A. Xu ${ }^{1,2,3}$, H.M. Zhong ${ }^{1,2}$ and Y.F. Zhang ${ }^{1,2}$ \\ ${ }^{1}$ Emergency Department, Second Affiliated Hospital, \\ Zhejiang University College of Medicine, Hangzhou, Zhejiang, China \\ ${ }^{2}$ Institute of Emergency Medicine, Zhejiang University, Hangzhou, \\ Zhejiang, China \\ ${ }^{3}$ Wound Healing and Cell Biology Laboratory, Burns Institute, \\ First Affiliated Hospital (304th Hospital), General Hospital of PLA, \\ Beijing, China \\ Corresponding author: Y.F. Zhang \\ E-mail: YufangZhangcn@126.com
}

Genet. Mol. Res. 14 (2): 2953-2957 (2015)

Received May 5, 2014

Accepted October 28, 2014

Published April 10, 2015

DOI http://dx.doi.org/10.4238/2015.April.10.3

\begin{abstract}
The purpose of this study was to analyze the therapy of a severe abdominal compartment syndrome (ACS) to elucidate the use of an abdominal advanced flap with other supportive measures for restoration of large defects of the abdominal wall. A patient presented with a large defect of the abdominal wall caused by ACS, which had resulted from multiple injuries after a fall from height. Healing of the defect was achieved by transplantation of an abdominal advanced flap and other supportive strategies. All of the treatment measures are presented to demonstrate complicated treatment procedures for closure of large defects of the abdominal wall. An abdominal advanced flap combined with other supportive measures was successfully applied in the healing process of ACS. This study examined the treatment of a case of ACS caused by severe abdominal trauma. The results demonstrated
\end{abstract}


that a large defect of the abdominal wall caused by ACS should be closed as early as possible, and an abdominal advanced flap combined with complex supportive measures can be a recommended strategy for closing large defects of the abdominal wall.

Key words: Abdominal compartment syndrome; Abdominal advanced flap; Intra-abdominal pressure; Laparotomy surgery decompression; Multiple trauma herniorrhaphy

\section{INTRODUCTION}

Abdominal compartment syndrome (ACS) is a severe complication of critically injured patients who sustain serious blunt abdominal trauma. Surgical decompression, along with delayed abdominal closure, is an effective strategy to treat the intra-abdominal hypertension (IAH) of ACS (van Mook et al., 2002; Cheatham, 2009; Urushidate et al., 2010). Once decompression is achieved, instant closure of the abdominal defect can substantially shorten the duration of serious symptoms. However, it is difficult to suture the abdominal wall primarily in some severe cases. Therefore, we have chosen alternative approaches, such as the abdominal advanced flap, to reconstitute the large defects of the abdominal wall caused by ACS. Generally, the front sheath of the rectus abdominis muscle needs to be separated to create a free fasciocutaneous flap for autogenous reconstruction of abdominal defects (Cheatham, 2009; Urushidate et al., 2010). The abdominal wall can then be completely sutured with lower tissue tension and present a better compliance to avoid the recurrence of ACS (Thorne et al., 2007). In the following case, we present a promising healing process that was successfully used to treat a patient with severe ACS.

\section{MATERIAL AND METHODS}

\section{Case report}

A 60-year old man was admitted for multiple injuries of the head, chest, abdomen, and limbs caused by an accidental fall from height. His main diagnoses were ACS, uncontrolled hemorrhagic shock, serious abdominal injuries, and injuries of other organs. The unstable hemodynamics, liver rupture, and damage of the pancreaticoduodenal duct directed us to conduct an immediate laparotomy, in which a "dome-like" incision was used to facilitate exploration of tissues destroyed. During the surgery, we repaired the damaged liver, ruptured diaphragm, and herniated omentum. Because the retroperitoneum and viscera were markedly swollen, closing the abdominal wall under high tissue tension could have been fatal for the patient (Figure 1A). Therefore, a sterile 3-L bag was used as a temporary device to close the large abdominal defect (Figure 1B).

Postoperatively, the patient was transferred to the intensive care unit (ICU) to receive supportive therapies and wound dressing treatment. Eighteen days later, his urinary output stabilized, which implied that it was an appropriate time to close the abdominal wound. When conducting the abdominal closure surgery, we found that retroperitoneal swelling and intestinal edema had not completely resolved, and the defect of the abdominal wound was as large as $25 \mathrm{~cm}$ at its maximal width. Moreover, exposed intestines were adhered to each other and 
protruded beyond the cutaneous surface (Figure 1C). Because of these difficulties, it was impossible to suture the abdominal wall completely. Thus, we chose an abdominal advanced flap for separation of the rectus sheath to construct a free fasciocutaneous flap with length of 10$15 \mathrm{~cm}$. This was sutured over the incision of the rectus muscle to close the abdominal cavity. After lifting the incised upper and lower flaps, we separated the rectus sheath and superficial fascia, harvested enough space to suture bilateral flaps, and closed the abdominal wound (Figure 1D). Seven days after the second surgery, the patient was sent back to the ICU to receive nutritional support and antibiotic treatment for restoration of damaged bowel. At that time, debridement and simple drainage by vacuum sealing drainage (VSD) were applied to remove necrotic tissue and exudation. Twenty-three days after the second surgery, the patient was transferred from the ICU back to the general ward (Figure 1E-F).

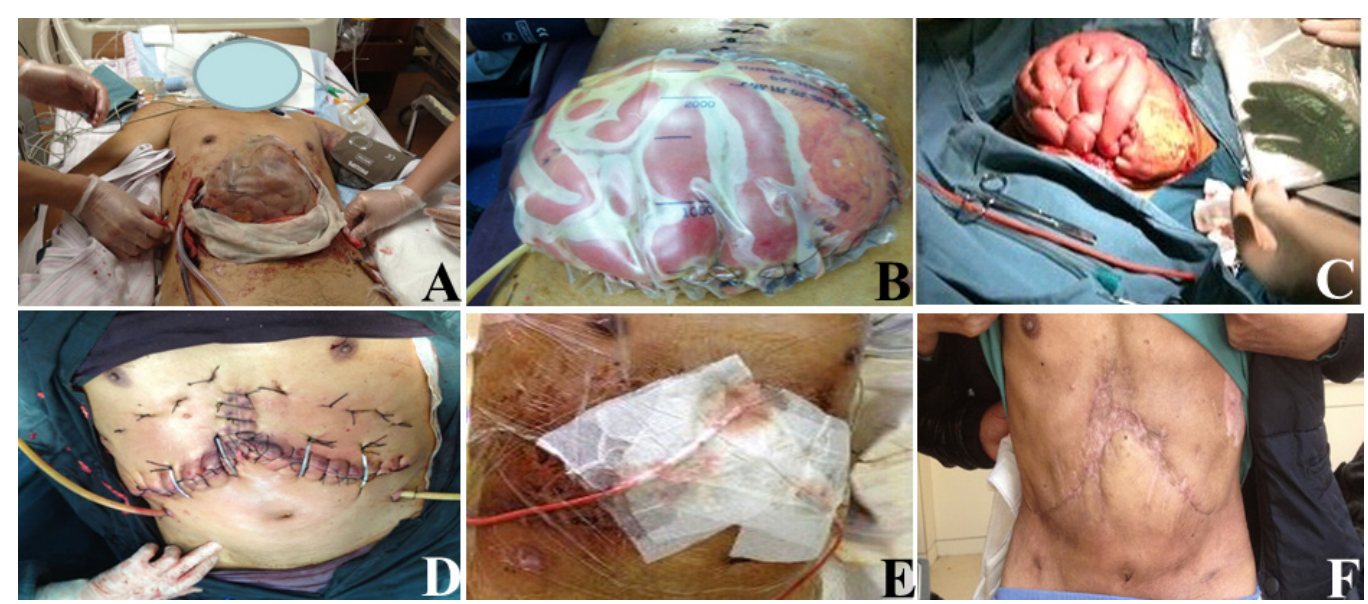

Figure 1. Appearance of serious ACS and its treatment process. A = temporary cover of large defect of ACS by a sterile 3-liters bag; $\mathbf{B}=$ swelled intestinal and large defect of abdomen; $\mathbf{C}=$ No infection in wound and abdominal cavity; $\mathbf{D}=$ coverage of ACS by abdominal flap; $\mathbf{E}=$ simple drainage of exudate and necrotic tissue by vacuum sealing attract (VSD); $\mathbf{F}$ = wound healing of large defect of abdominal wall.

\section{DISCUSSION}

Surgical decompression or delay in closing the abdomen are generally recognized as effective measures for ACS (Balogh et al., 2007; Vidal et al., 2008; Björck, 2012; Demetriades, 2012). However, when and how to close the abdominal defect are difficult for surgeons in the emergency department to determine. Currently, widely accepted indications for when to attempt closure are as follows (Vidal et al., 2008; Björck, 2012; Demetriades, 2012): reduction of intra-abdominal pressure to a normal level, stability of hemodynamics, increase in urinary output, resolution of edema, and correction of coagulopathy. The opportunity for closure usually occurs approximately two weeks after surgery, but may occur later for severe ACS patients. Prolonged exposure of wounds, especially in patients with multiple trauma and serious abdominal injury, can result in loss of protein, electrolyte disorders, and wound and intra-abdominal infections (Vidal et al., 2008). Because the above indicators for abdominal closure may never be completely achieved, it has been proposed to close the abdominal cavity as soon as possible, provided that hemodynamics and urine output are stable and intra-abdom- 
inal pressure is reduced, especially when the postoperative abdominal exposure time has been longer than 14 days. If intestinal edema has not completely resolved, an abdominal advanced flap is strongly suggested to replace the primary sutures. We separated the superficial fascia with an incision of the rectus and constructed a free fasciocutaneous flap to directly cover the bowel surface (Vidal et al., 2008; Demetriades, 2012). The advantages of this technology include the following: 1) to avoid a recurrence of ACS caused by IAH; 2) to temporarily expand the abdominal cavity to contain the intestines and close the abdominal wound; 3) to reduce the recurrence of ACS through the retaining fascia without suturing the rectus; 4) to increase feasibility of isolation of the skin flap from the front of the rectus sheath and muscle because of the thickened abdominal wall and clearly separated layers; and 5) to easily supplement the flap with a prosthetic patch to close the abdominal cavity if the free flap cannot be completely sutured. Moreover skin flaps have several advantages when compared with skin transplantation: 1) a more abundant blood supply in skin flaps vs skin grafts helps avoid skin failure; 2) the thicker tissue of skin flaps more effectively protects the bowel from developing a fistula; and 3) skin flaps combined with VSD can achieve better drainage of necrotic tissue and exudation. Skin flaps can maintain a stable intraperitoneal environment, reduce the risk of intestinal fistula, help recovery of intestinal function, and facilitate entry of nutrition by establishment of an autogenous environment. The procedures described provide a foundation for rehabilitation of severe multiple trauma patients. Once patients pass the critical stage of ACS, herniorrhaphy and other abdominal surgical techniques can completely repair abdominal wall defects (van Mook et al., 2002; Demetriades, 2012).

Based on our limited experience, a large defect of the abdominal wall caused by ACS should be closed as early as possible, and an abdominal advanced flap, combined with complex supportive measures, might be a recommended strategy for closing large defects of the abdominal wall.

\section{ACKNOWLEDGMENTS}

Research supported partially by the National Natural Science Foundation of China (Grant \#81201478, \#81372079), the Specialized Research Fund for the Doctoral Program of Higher Education (Grant \#20120101120035), the Research Special Fund for Public Welfare Industry of Health (\#20100 \#2014), the Fund of Zhejiang Innovative Research Group (Grant \#2011R50018), and the State Key Development Program of Basic Research of China ("973" Program, Grant \#2012CB 5181 05).

All authors have contributed to the treatment of the patient and the writing of the manuscript. The authors declare no conflicts of interest.

\section{REFERENCES}

Balogh Z, Moore FA, Moore EE and Biffl WL (2007). Secondary abdominal compartment syndrome: A potential threat for all trauma clinicians. Injury 38: 272-279.

Björck M (2012). Management of the tense abdomen or difficult abdominal closure after operation for ruptured abdominal aortic aneurysms. Semin. Vasc. Surg. 25: 35-38.

Cheatham ML (2009). Nonoperative management of intraabdominal hypertension and abdominal compartment syndrome. World J. Surg. 33: 1116-1122.

Demetriades D (2012). Total management of the open abdomen. Int. Wound J. 9 (Suppl 1): 17-24.

Thorne CH, Beasley RW, Aston SJ, Bartlett SP, et al. (2007). Grabb and Smith's Plastic Surgery (6th edn). Lippincott 
Williams \&Wilkins, Philadelphia, pp. 670-675.

Urushidate S, Yotsuyanagi T, Yamauchi M, Mikami M, et al. (2010). Modified thin abdominal wall flap (glove flap) for the treatment of acute burns to the hands and fingers. J. Plast. Reconstr. Aesthet. Surg. 63: 693-699.

van Mook WN, Huslewe-Evers RP and Ramsay G (2002). Abdominal compartment syndrome. Lancet 360: 1502.

Vidal MG, Ruiz Weisser J, Gonzalez F, Toro MA, et al. (2008). Incidence and clinical effects of intra-abdominal hypertension in critically ill patients. Crit. Care Med. 36: 1823-1831. 